\section{Explorative study on the use of omalizumab in patients suffering from interstitial cystitis/bladder pain syndrome}

\author{
Daniele Porru, ${ }^{1}$ Enrica Bucchioni, ${ }^{2}$ \\ Marcello Macchi, ${ }^{1}$ Fabio Leva, ${ }^{1}$ \\ Alberto Parmigiani, ${ }^{1}$ Davide Barletta, ${ }^{1}$ \\ Dimitrios Choussos, ${ }^{1}$ Barbara Gardella, ${ }^{3}$ \\ Maria Diletta Daccò, ${ }^{3}$ \\ Rossella Elena Nappi, ${ }^{3}$ Massimo Allegri, ${ }^{4}$ \\ Carlo Maria Bianchi, ${ }^{5}$ Arsenio Spinillo, ${ }^{3}$ \\ Bruno Rovereto' \\ 'Urology Department, IRCCS San Matteo \\ Hospital, Pavia, Italy; ${ }^{2}$ Novartis Italia, \\ Origgio (VA), Italy; ${ }^{3}$ Obstetric and \\ Gynecology Clinics IRCCS San Matteo \\ Hospital, Pavia, Italy; ${ }^{4}$ Pain Therapy \\ Service Fondazione IRCCS Policlinico San \\ Matteo, Department of Resuscitation and \\ Organ Transplantation surgery sciences, \\ Section of Anesthesiology and \\ Resuscitation, University of Pavia, Italy; \\ ${ }^{5}$ School of Specialization in Urology, \\ University of Pavia, Italy
}

\section{Abstract}

The aim of this study was to evaluate the efficacy of omalizumab in the treatment of Interstitial Cystitis/Bladder Pain Syndrome (IC/BPS), evaluated by visual analogue score for pain and urgency- frequency, 0'Leary-Sant IC symptom and problem index questionnaire, Pain Urgency Frequency questionnaire and Patient Global Assessment questionnaire. Four female patients with a diagnosis of IC/BPS were included in the study, with an age between 20 and 39 years. In the first patient the subjective final evaluation was of a marked improvement. The second patient had a moderate improvement of the subjective final evaluation. The third patient considered her overall clinical situation to have slightly improved after treatment. One 32-year-old patient, with multiple allergies, discontinued treatment after 3 months and could not complete the study due to side effects. Omalizumab was subcutaneously administered to patients with IC/PBS; it induced both a subjective and objective improvement of symptoms in 2 patients enrolled in the study.

\section{Introduction}

The bladder symptom complex interstitial cystitis/bladder pain syndrome (IC/BPS) includes a large group of patients with bladder and/or urethral and/or pelvic pain, lower urinary tract symptoms and sterile urine cultures, many with specific identifiable causes. IC/BPS comprises a part of this complex. The IC/BPS definition is used, agreed upon by the Society for Urodynamics and Female Urology: ${ }^{1}$ An unpleasant sensation (pain, pressure, discomfort) perceived to be related to the urinary bladder, associated with lower urinary tract symptoms of more than six weeks duration, in the absence of infection or other identifiable causes. ${ }^{2}$

In some cases of IC/BPS a urogenital disorder of allergic nature has been hypothesized. ${ }^{3-5}$ Morphological studies have demonstrated an increase in the number and activation of mast cells in IC/PBS. ${ }^{4}$ Yamada et al. ${ }^{6}$ reported an incidence of $80 \%$ of allergic disorders in 34 patients diagnosed with IC/BPS. In a patient suffering from IC/BPS and chronic urticaria-angioedema, bladder biopsy showed mastocytosis with cell degranulation. It has also been reported that treatment with omalizumab in a 28 years old female patient with IC showed complete clearance of the genitor-urinary condition, with posttreatment cystoscopic evaluation demonstrating a reduction in inflammatory infiltrates and the disappearance of Hunner ulcers. ${ }^{3}$

By hypothesising that in some cases IC/BPS may be an allergic disorder of the urogenital system linked to mast-cells, therapy with omalizumab may represent a potential non symptomatic strategy for the treatment of this disease. It has been approved by FDA for its use in adult patients and in adolescents over 12 years of age with moderate-to severe persistent allergic asthma that is not adequately controlled, despite inhalation therapy with corticosteroids. ${ }^{7,8}$

The objective of this explorative study was to evaluate the efficacy of omalizumab in the treatment of IC/BPS, evaluated by visual analogue score (VAS) for pain and urgency- frequency $\left(\mathrm{VAS}_{\mathrm{p}}\right.$ and $\mathrm{VAS}_{\mathrm{u}}$ ), 0'Leary-Sant IC Symptom Index/Problem Index (ICSI-ICPI) (primary outcome), Pain Urgency Frequency (PUF) questionnaire and Patient Global Assessment questionnaire (secondary outcomes).

Data derived from registered clinical trials and from post-marketing surveillance confirm that omalizumab is a safe and well-tolerated drug. It has been approved by FDA in June 2003 for its use in adult patients and in adolescents over 12 years of age with moderate-to severe persistent allergic asthma that is not adequately controlled, despite inhalation therapy with corticosteroids. Since then, more than 90,000 patients have been treated all over the world. The most common side effects of omalizumab (observed in $0.1-10 \%$ of patients) are headache and local reactions at the site of administration, which include pain, swelling, erythema and itching. Omalizumab should not be used in individuals that are potentially
Correspondence: Daniele Porru, Divisione Urologia, Fondazione IRCCS Policlinico San Matteo, viale Golgi 19 , Pavia 27100 , Italy.

Tel. $+39.0382 .503 .868 / 503.872$

Fax: +39-0382.503.403

E-mail: danieleporru@tin.it

Key words: anti-IgE treatment, interstitial cystitis/bladder pain syndrome, omalizumab.

Trial registration: ClinicalTrials.gov number NCT01294878.

Contributions: DP, EB, protocol/project development; MM, FL, AP, DB, DC, data collection or management; BG, MDD, REN, MA, data analysis; $\mathrm{CMB}, \mathrm{AS}, \mathrm{BR}$, manuscript writing/editing.

Funding: Novartis Italia provided the drug for all patients treated, and sustained the costs of the examinations involved in the study.

Received for publication: 8 August 2011.

Revision received: 11 August 2011.

Accepted for publication: 11 May 2012.

This work is licensed under a Creative Commons Attribution NonCommercial 3.0 License (CC BYNC 3.0).

CCopyright D. Porru et al., 2012

Licensee PAGEPress, Italy

Urogynaecologia 2012; 26:e8

doi:10.4081/uij.2012.e8

hypersensitive (allergic) to the active compound or to other components of the drug.

\section{Materials and Methods}

Four female adult patients aged between 20 and 39 years, suffering form IC/BPS, having failed oral (pentosanpolisulphate) and intravesical treatment (hyaluronic acid) previously administered (except patient 1), were included in the study. They were not consecutive, since had total serum IgE between 30 and 700 IU/mL, and had a positive allergic anamnesis, and asthma, rhinitis, atopic dermatitis were present at inclusion in the study.

One 32-year-old patient, with multiple allergies, discontinued treatment after 3 months and was excluded from the study due to side effects including general discomfort, nausea and dyspnoea, which lasted 7-10 days during assumption of treatment. Interruption of treatment resulted in complete recovery; it is likely that intolerance to omalizumab had developed.

\section{Inclusion criteria}

i. Female patients $\geq 18$ years, with a range in body weight of $\geq 20$ and $\leq 150 \mathrm{~kg}$, who have 
provided written informed consent;

ii. in patients diagnosed with IC/BPS that underwent treatment, basal levels of total serum IgE were $\geq 30$ and $\leq 700 \mathrm{IU} / \mathrm{mL}$ and patients showed a positive allergic anamnesis (asthma, rhinitis, atopic dermatitis, etc.);

iii. diagnosis of IC/BPS was performed according to the criteria established by the National Institute of Health Consensus Conference $^{1}$ and patients had symptoms for at least 6 months. Cytoscopy was performed according to the clinical indications.

\section{Exclusion criteria}

i. Pregnancy, breastfeeding. Fertile women that did not use secure contraceptive methods (hormonal or double barrier method). Hysterectomized or surgically sterilized women (tubal ligation) and menopause women were admitted into the study;

ii. clinically relevant medical conditions (neoplasia, infections, hematologic, renal, hepatic, cardiovascular, hormonal or gastrointestinal pathologies) within 3 months prior to the study. Other specific criteria included patients with positive anamnesis for bladder cancer or affected by actinic cystitis, vaginitis, symptomatic bladder or urethral diverticulum, active genital herpes, bladder or urethral lithiasis;

iii. urination frequency $<5$ times per day;

iv. known hypersensitivity to any omalizumab component, excipients included (such as monoclonal antibodies, polyclonal gamma globulins);

v. alcohol or drug abuse.

The study had Institutional Review Board approval, written informed consent was obtained from all patients prior to participation in the study and establishing therapy administered during the run-in period. A 4-week run-in period was performed and a subsequent treatment period (48 weeks) was followed with 4 medical visits, every 3 months.

The omalizumab dose has been calculated on the basis of body weight and basal levels of total serum IgE. Treatment was administered subcutaneously every 2 or 4 weeks (according to the calculated total dose) for a total of 48 weeks. Each vial contained $150 \mathrm{mg}$ of the active compound, therefore the number of injections for each administration varied between 1 and 3 , depending on body weight and basal levels of total serum IgE. Patients were allowed to take drugs used for IC/BPS: oral Pentosanpolysulphate $100 \mathrm{mg} 3$ times per day, Pelvilen $440 \mathrm{mg} 2$ times per day, oral Quercetin-Chondroitin sulphate-Glucosamine sulphate $650 \mathrm{mg} 2$ times per day.

This is important given that it is expected that treatment is part of multimodal therapy; during the screening process, the dosage of these drugs was established and stably main- tained during the 4-week run-in period.

Topical therapies (such as intravesical instillation, intravesical electromotive, botulinum toxin injections) or pudendum nerve blocks and S3 sacral neuromodulation were not allowed.

\section{Efficacy evaluation}

The efficacy variable was defined as the response to treatment during the 12-month therapy with omalizumab, and it was evaluated by the following methods: ${ }^{9}$

i. VAS for pain and urgency-frequency;

ii. a 3-day urination diary;

iii. 0'Leary-Sant (ICSI-ICPI) and PUF questionnaires at 2 months prior to the study, time 0 (baseline) and every 3 months thereafter;

iv. patient Global Assessment.

\section{Safety evaluation}

To evaluate safety and tolerability of the drug, patients were monitored during each medical inspection for side effects and severe collateral effects. Laboratory tests included total IgE before initiation and after treatment, urine examination and urine culture.

Three out of four IC/BPS patients completed the study without manifesting side effects due to treatment. Patients' age was between 20 and 39 years, all of them had history of allergy consisting in seasonal allergies or referred allergy to drugs, and were otherwise healthy. Prior to the study P.R. underwent laparohysterectomy due to uterine fibromatosis.

\section{Results}

\section{Patient \#1 (D.D.)}

D.D., aged 20 years, was included in the study immediately following diagnosis that was established following a relatively short clinical history and with symptoms mostly represented by bladder pain (basal VAS 10/10) accompanied by urgency (basal VAS 5/10). Urinary frequency was not significantly increased. VAS values of both pain and urgency were reduced to 1 and 0 , respectively, when evaluated at the first trimester after starting treatment, and remained unchanged until treatment completion 12 months after (Figure 1). 0'Leary ICSI-
ICPI questionnaire varied from basal levels of 7 to 4 at the end of therapy (Figure 2). The patient global assessment was of a marked improvement. The patient has been re-assessment 6 months after the end of treatment, showing stable, persistent improvement.

Total IgE levels were $328 \mathrm{IU} / \mathrm{mL}$ and 1060 IU/mL, respectively before and after 12 months treatment (Table 1).

\section{Patient \#2 (C.Z.)}

C.Z., aged 38 years, showed a reduction in bladder pain, with a VAS varying from $4 / 10$ to $2.8 / 10$ in the second trimester and to $2 / 10$ at the end of the treatment period (12 months). It was not possible to observe a similar effect on urgency, as this parameter was not clinically relevant at the time the patient was enrolled in the study, with a basal VAS of $0.7 / 10$. A VAS value of $0 / 10$ was scored at the third trimester and at the end of the study (Figure 1). For this patient, the overall 0 'Leary symptom score varied from 18 to 12 , and this was observed in the fourth trimester of therapy. In addition, the 0'Leary ICSI-ICPI score, divided into symptom and psychological impact components revealed an improvement in the first category (18 to 12 , final value), while the problem index appeared largely unchanged, with a score varying from 7 to 6 . The patient global assessment was of a moderate improvement.

Total IgE levels were $146 \mathrm{IU} / \mathrm{mL}$ and 230 , $\mathrm{IU} / \mathrm{mL}$ respectively before and after 12 months treatment.

\section{Patient \#3 (P.R.)}

In P.R., aged 39 years, a modest improvement of symptoms could be observed during the treatment course, with an 0'Leary symptom score varying from 23 to 20 after 12 months (Figure 1). VAS values of urgency varied from 4.3 to 3.6, while bladder pain VAS was increased from 3.1 to 6.2 . The patient global assessment was of slight improvement. In this case, the treatment employed was a multimodal one and the patient followed an analgesic therapy approach. PUF questionnaire scores are reported in Figure 3.

Total IgE levels were $258 \mathrm{IU} / \mathrm{mL}$ and 696 $\mathrm{IU} / \mathrm{mL}$, respectively before and after 12 months treatment (Table 1).

Table 1. Serum IgE values at baseline and after treatment.

\begin{tabular}{lcc}
\hline Patients' initials & Basal IgE & 12 months $\operatorname{IgE}$ \\
R.P. & 328 & 1060 \\
D.D. & 146 & 230 \\
\hline Z.C. & 258 & 696 \\
\hline
\end{tabular}




\section{Discussion}

Since Keay ${ }^{9,10}$ postulated the presence of the anti-proliferative factor in the IC/PBS and investigated the role played by this protein in the aetiology of this disease, no major findings have been discovered on aetiology and pathogenesis of IC/BPS. In two women with a long history of bladder symptoms refractory to any urologic therapy administered before and with allergic rhinitis and multiple arthralgies, ${ }^{11}$ a casual participation of inhaled allergens and of diet components to the onset of urinary symptoms was observed. By removing these allergic agents and by orally administering disodiocromoglicate an almost complete remission of urinary and non-urinary symptoms could be observed. This indicates that only seldom allergic pathologies are considered to participate in the development or are included in the differential diagnosis of this disease.

Undoubtedly, epidemiological studies show a higher prevalence of allergies in IC/BPS patients compared to general population, ${ }^{9}$ in addition anti allergic drugs can exert a positive effect on bladder symptoms, particularly in the presence of seasonal allergies. ${ }^{12}$

Vulvodynia is frequently associated with IC/BPS ${ }^{13,14}$ and allergy also seems to play a role in this condition, ${ }^{15}$ which involves both the sexual and the urinary spheres. Thus, the link between allergy, IC/BPS and vulvodynia does not seem to be by chance. A Japanese study ${ }^{6}$ shows that complications of allergic diseases are mostly frequent in young IC/BPS patients, and that $80 \%$ of patients with IC/PBS experience allergic manifestations.

Omalizumab is a recombinant humanized monoclonal antibody that selectively binds to circulating human IgE and blocks the IgE-mediated allergic reaction, by preventing IgE adhesion to effectors cells. Moreover, omalizumab negatively modulates the expression of $\operatorname{IgE}$ receptors, as a direct consequence of the reduction in free circulating IgE. Omalizumab is a non-anaphylactic antibody, because its binding site is positioned on the same portion of $\operatorname{IgE}$ that binds the receptor, while it does not recognize IgE antibodies already bound to cell receptors. Omalizumab-IgE complex are small and are unable to activate the complement, thus preventing toxicity complement-dependent.

Free IgE and IgE complexed by Omalizumab actually rise under Omalizumab treatment, whereas levels of free IgE alone markedly and rapidly (within a few hours after the first Omalizumab application) decrease. This may explain why levels of total (free and complexed) IgE were increased in all patients at the end of treatment.

of note, the young patient that experienced the most significant beneficial effect from the therapy and that still maintains these effects even after treatment suspension, underwent exams to diagnose IC/PBS immediately prior to starting omalizumab therapy, following a short clinical history of the disease (a few months). Therefore, this has been the first long term therapy she has undertaken, with a very short interval between treatment and diagnosis.

We could speculate that if interstitial cystitis were a disease in which IgE specific to auto antigens/allergens played a major role, the beneficial effects of Omalizumab should also vanish some time after discontinuation of the drug since the causative auto-antigens are still present and should boost specific IgE-production. However, a possible mechanism compatible with this hypothesis maybe that these auto-antigens are not exposed any more after remission of the lesions in the bladder (corre-

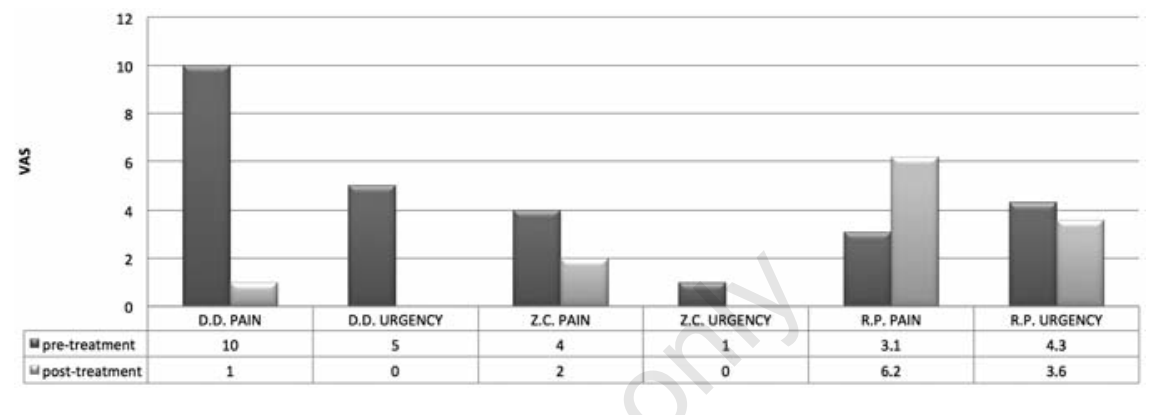

Figure 1. Patients' visual analogue score (VAS) score before and after treatment in the 3 patients.

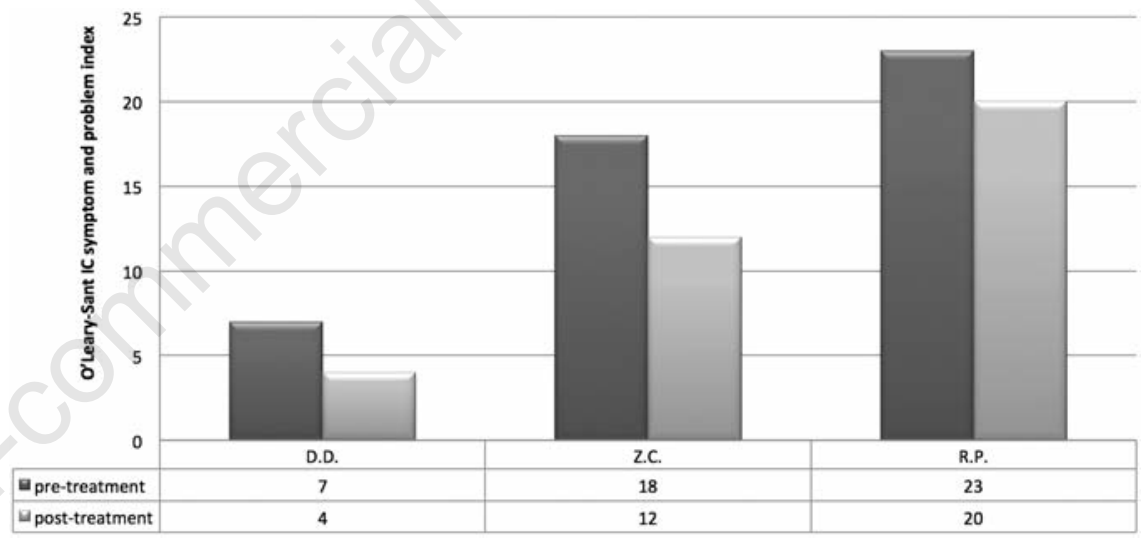

Figure 2. Trends of O'Leary-Sant interstitial cystitis (IC) symptom and problem index in the 3 patients.

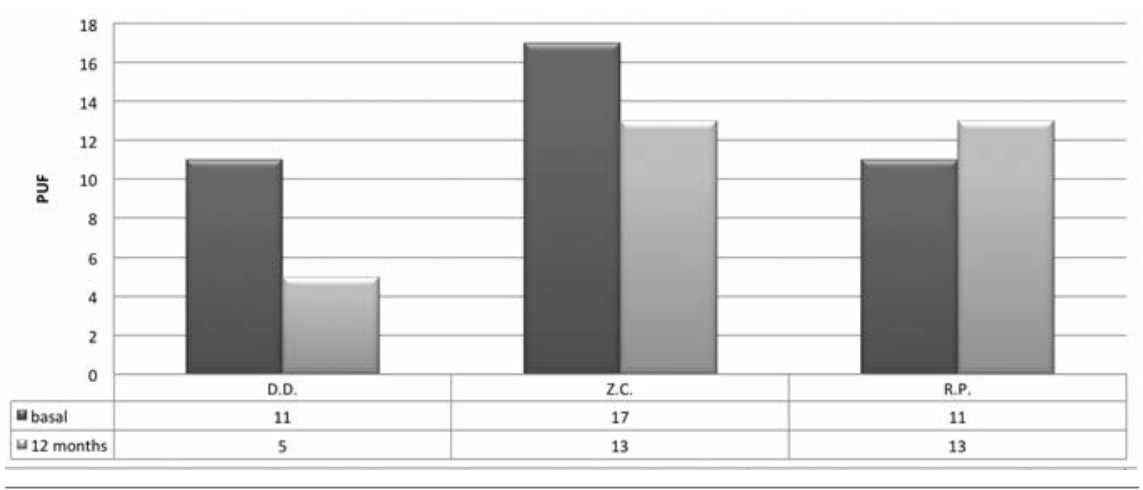

Figure 3. Score of pain urgency frequency (PUF) questionnaire in the 3 patients before and after treatment. 
sponding to theories on atopic dermatitis in which the release of auto-antigens upon scratching has been proposed to trigger inflammation in the skin). We understand that in a small study on a drug that acts through an immunological mechanism related to mast cells and IgE, performing a cystoscopy and biopsy would have been useful despite the fact that such an evaluation is not otherwise mandatory for the treatment of PBS-IC. This might have given some objective assessment of the effect of the drug, although represents a burden from patients' perspective.

\section{Conclusions}

In this explorative study, the monoclonal anti-IgE antibody omalizumab was subcutaneously administered to patients with IC/BPS and showed to induce both a subjective and objective improvement of symptoms in 2 out of the 3 patients enrolled in the study. An adequate population of IC/PBS patients, in which an allergic disorder coexists with urinary symptoms, could provide a satisfactory response to the omalizumab treatment. Certainly the number of patients should be increased, and due to the well-known placebo effect a placebo arm should be considered in a future representative clinical trial to evaluate the effects of such anti-allergic therapy, in terms of safety, efficacy and interaction with other drugs.

\section{References}

1. Hanno PH, Burks DA, Clemens JQ, et al. Guideline for the diagnosis and treatment of interstitial cystitis/bladder pain syndrome. J Urol 2011;185:2162-70.

2. Hanno P, Dmochowski R. Status of international consensus on interstitial cystitis/bladder pain syndrome/painful bladder syndrome: 2008 snapshot. Neurourol Urodyn 2009;28:274.

3. Lee J, Doggweiler-Wiygul R, Kim S, et al. Is interstitial cystitis an allergic disorder?: A case of interstitial cystitis treated successfully with anti-IgE. Int J Urol 2006;13:6314.

4. Ochcs RL, Stein TW Jr, Peebles CL, et al. Autoantibodies in interstitial cystitis. J Urol 1994;151:587-92.

5. Denson MA, Griebling TL, Cohen MB, Kreder KL. Comparison of cystoscopic and hystological fundings in patients with suspected interstitial cystitis. J Urol 2000;164: 1908-2011.

6. Yamada T. Significance of complications of allergic diseases in young patients with interstitial cystitis. Int J Urol 2003;10 Suppl:S56-8.

7. Holgate ST, Chuchalin AG, Hebert J, et al. Efficacy and safety of a recombinant antiimmunoglibulin E antibody (omalizumab) in severe allergic asthma. Clin Exp Allergy 2004;34:632-8.

8. D'Amato G, Bucchioni E, Oldani V,
Canonica W. Treating moderate-to-severe allergic asthma with a recombinant humanized anti-IgE monoclonal antibody (Omalizumab). Treat Respir Med 2006;5: 393-8.

9. Pelikan Z, van Oers JA, Levens WJ, Fouchier SM. The role of allergy in interstitial cystitis. Ned Tijdschr Geneeskd 1999;143:1289-92.

10. Keay S, Warren JW. A hypothesis for the etiology of interstitial cystitis based upon inhibition of bladder epithelial repair. Med Hypotheses 1998;51:79-83.

11. Keay SK, Szekely Z, Conrads TP, et al. An antiproliferative factor from interstitial cystitis patients is a frizzled 8 proteinrelated sialoglycopeptide. Proc Natl Acad Sci USA 2004;101:11803-8.

12. Zámecník L, Hanus T, Pavlík I, et al. Statistical analysis of symptoms, endoscopy and urothelial morphology in 58 female bladder pain syndrome/interstitial cystitis patients. Urol Int 2009;83:193-9.

13. Theoharides TC, Sant GR. Hydroxyzine therapy for interstitial cystitis. Urology 1997;49(5A Suppl):108-10.

14. Gardella B, Porru D, Ferdeghini F, et al. Insight into urogynecologic features of women with interstitial cystitis/painful bladder syndrome. Eur Urol 2008;54:114551.

15. Haefner HK, Collins ME, Davis GD, et al. The vulvodynia guidelines. J Low Genit Tract Dis 2005;9:40-51. 